\title{
Generalism and the Metaphysics of Ontic Structural Realism*
}

\author{
David Glick \\ david.glick@philosophy.ox.ac.uk
}

\begin{abstract}
Ontic structural realism (OSR) claims that all there is to the world is structure. But how can this slogan be turned into a worked-out metaphysics? Here I consider one potential answer: a metaphysical framework known as generalism (Dasgupta, 2009, 2016). According to the generalist, the most fundamental description of the world is not given in terms of individuals bearing properties, but rather, general facts about which states of affairs obtain. However, I contend that despite several apparent similarities between the positions, generalism is unable to capture the two main motivations for OSR. I suggest instead that OSR should be construed as a meta-metaphysical position.
\end{abstract}

\section{Introduction}

Structural realism in the philosophy of science maintains that our best scientific theories tell us only about the structure of the world, not the full complement of objects and properties recognized by traditional realists. Ontic structural realism (OSR) goes further and claims that all there is to the world is structure. Despite its popularity among philosophers of physics, many remain skeptical of OSR. What does it mean to say that "structure is all there is"? How can this slogan be turned into a worked-out metaphysics?

One potential answer is the metaphysical framework of generalism advanced by Shamik Dasgupta $(2009 ; 2016)$. According to the generalist, the most fundamental description of the world is not given in terms of individuals bearing properties, but rather, general facts about which states of affairs obtain. This framework allows the advocate of OSR to state the fundamental facts without being committed to the existence of any individual objects. However, I will argue below that generalism is inadequate as an explication of OSR. As a version of structural realism, OSR requires making a concession to the antirealist; much of the content endorsed by traditional realists must be jettisoned to make sense of theory change. A generalist metaphysics does no better with respect to this requirement, and may even do worse than traditional realism. Generalism appears better suited to capture another motivation for OSR, namely, offering an explanation of permutation invariance in

\footnotetext{
${ }^{*}$ Forthcoming in The British Journal for the Philosophy of Science.
} 
quantum theory. Even in this case, though, generalism fails to achieve the structuralist's aims.

Several conclusions might be drawn from this. First, one may come to regard OSR as simply untenable, or as collapsing into a more standard form of realism or antirealism (Psillos, 2006). Second, one might hope for an alternative metaphysical framework for OSR. Below I will suggest another option: to understand OSR as a meta-metaphysical thesis rather than a particular first-order metaphysical position. On this view, OSR is (roughly) the idea that mathematical models provide the best representations of physical reality. I contend that such an understanding captures the core motivations of OSR better than generalism, or indeed, than any rival first-order metaphysical view is likely to. While I stop short of endorsing this version of OSR, it seems to me to represent the best way for structuralists to develop their view.

\section{Motivations}

OSR draws motivation from two main paths: the problem of theory change in the philosophy of science and various interpretative issues in modern physics. We will briefly consider the former and a common theme among instances of the latter.

\section{$2.1 \quad$ Theory change}

Why should we believe our current best scientific theories to be correct when all of our past theories have proven to be incorrect? This is a version of the pessimistic meta-induction argument against scientific realism. The typical response from realists is to retreat to approximate truth. We shouldn't think that our current theories have got things exactly right, but just that they are on the right track. However, as Laudan (1981) famously argues, this doesn't obviously help. A plausible necessary condition for our current theories being approximately true is that they get the ontology right, but there appear to be several successful past theories that were incorrect about ontology; heat isn't a fluid; light isn't a disturbance in luminiferous ether; there is no phlogiston, etc. The problem for the realist is that, as Kuhn observed, there is no continuity in the development of scientific theories at the level of ontology $(\overline{\mathrm{Kuhn}}, 1996,206)$.

However, if we change our focus from ontology to structures, continuity can be restored. Or, at least, so claims Worrall (1989) and structural realists following him. To use a wellworn example from the history of optics, throughout the changes in our understanding of what light $i s$, there has been continuity in how light behaves. This continuity is evidenced by the fact that Fresnel's equations describing displacements in luminiferous ether can be derived from Maxwell's equations of the electromagnetic field (cf., Saatsi, 2005). The structural realist, then, claims that we are justified in believing in the structure of our current best theories because it is likely that these structures will be continuous with those posited by future theories. 
If successful, this line of reasoning supports structural realism as an epistemic stance: we should endorse only the structural content of our best theories. How do we get from here to OSR? ${ }^{1}$ There are several arguments, but the simplest is based on a kind of methodological naturalism. If we grant that science should be our guide to reality, and we accept that on its basis we can only know the structure of the world, then we should only posit structure. On this way of thinking, the problem with epistemic structural realism (ESR) is that it creates an unmotivated gap between epistemology and metaphysics; there is simply no reason to think there is more to the world than we can possibly know (Ladyman and Ross, 2007, 154) ${ }^{2}$

\subsection{Permutation invariance}

There are several issues in the philosophy of physics that structuralists have taken to support OSR. One general theme concerns how physics treats permutations of basic objects. For example, systems of indistinguishable particles in quantum theory display permutation invariance; states related by a permutation of particles (or particle labels) are taken to represent the same physical state of affairs ${ }^{3}$ A related feature in the context of spacetime theories is diffeomorphism invariance in general relativity. The dominant view there is that diffeomorphic models - those related by a smooth continuous shift in the metric and matter fields - are physically equivalent. I will focus on the case of quantum particles to demonstrate the argument for OSR, but analogous reasoning has been used to advocate spacetime structuralism 4

Consider two indistinguishable particles in an entangled state that is a superposition of $A_{1} B_{2}$ and $B_{1} A_{2}$, where $A$ and $B$ are one-particle states and 1 and 2 represent distinct particles 5 On the orthodox understanding of such a state, one cannot regard 1 or 2 as being in either state $A$ or $B$, but can be sure that upon measurement they will be found in different states. Moreover, permuting 1 and 2 doesn't seem to make any empirical difference; we could have just as easily written the superposition with the particle labels in the opposite order. There are many subtleties to the issue of permutation invariance, and related issues in quantum statistics, but the take away lesson for the proponent of OSR is as follows: elementary particles of the same kind may be permuted without generating a

\footnotetext{
${ }^{1}$ Ladyman (1998) was the first to articulate OSR and defend it over epistemic versions of structural realism.

${ }^{2}$ Perhaps the best known argument against ESR is based on Newman's objection to Russell's 1927 version of structuralism. There is an extensive literature on this topic and reviewing it here would take us too far afield (for an overview, see Ladyman (2009, §3.2)).

${ }^{3}$ Discussions of permutation invariance and structuralism include French and Redhead (1988); French (1989); Ladyman and Ross (2007); Caulton and Butterfield (2012); Saunders (2015).

${ }^{4}$ For attempts to connect permutation invariance and diffeomorphism invariance in context of structuralism see Stachel (2002); French and Rickles (2003); Glick (2015), although, see also Pooley (2006).

${ }^{5}$ For instance, a pair of spin $\frac{1}{2}$ particles in the spin singlet state: $\psi=\frac{1}{\sqrt{ } 2}(|\uparrow\rangle \otimes|\downarrow\rangle-|\downarrow\rangle \otimes|\uparrow\rangle)$, where $\uparrow$ and $\downarrow$ indicate spin values of $+\frac{1}{2}$ and $-\frac{1}{2}$, respectively, along some arbitrary axis.
} 
new state of affairs.

What does this have to do with OSR? Again, there are several ways of connecting the issues, but the central point is clear: only the structure of the situation seems to matter and, as a result, we shouldn't think of the particles involved as objects that exist "over and above" structure. Let's unpack this a bit. If we have two objects that could be in two states, we would ordinarily think that there are four possible configurations. But, this runs afoul of permutation invariance as two of these configurations are related by a permutation of the basic objects (or their labels). How should we view these objects instead? If they are somehow dependent on the structure of the situation, then we get the desired result; permutations don't matter because they preserve the structure of the situation.

The metaphysical underdetermination argument of French and Ladyman (2003) seeks to draw a stronger conclusion from these issues. The initial premise of the argument is that the nature of elementary particles is underdetermined by physics. Permutation invariance, and quantum statistics more generally, is compatible with both an understanding of particles as individuals and one in which they are non-individuals ${ }^{6}$

Given that the metaphysical nature of particles is underdetermined in this way, French and Ladyman argue that we should eliminate them from our ontology in favor of structures; if we can't say what kind of entities elementary particles are, we ought not commit ourselves to their existence. There has been considerable resistance to this argument, even among structuralists (e.g., Brading and Skiles (2012)), but the general point doesn't require it. Those who find support for OSR in permutation invariance claim that, in some sense, permutations are blocked by adopting a structuralist metaphysics.

\section{Metaphysics}

OSR is typically construed as an attempt to replace the traditional ontology of objects and (monadic) properties with an ontology of structure alone. Yet, common understandings of structure seem to require objects. The set-theoretic understanding of structure is simply a domain of objects with relations defined over them:

$$
\mathcal{S}={ }_{d f}\langle D, R\rangle
$$

But, given such an understanding, it's incoherent to suppose that the world has a certain structure without containing objects.7 More informally, structure is sometimes described as a network of relations. But, the usual way of understanding a relation also seems to require objects to be instantiated.

\footnotetext{
${ }^{6}$ It's not entirely clear what is meant by "individual" and "non-individual" in this context, but roughly an individual is an object with discerning identity conditions - e.g., a unique determinate trajectory in spacetime - and a non-individual lacks such a means of individuation. This notion of an individual should be distinguished from Dasputa's use below, although the two are no doubt related.

${ }^{7}$ One way to avoid this problem would be to adopt an alternative formal framework, such as category theory (Bain, 2011). However, see Lam and Wüthrich (2015) for challenges.
} 


$$
R a b \vDash \exists x \exists y R x y
$$

In other words, relations require relata, and hence "relational structure" requires objects as well. Thus, OSR seems to require a different understanding of structure from these familiar ones. At the very least, more needs to be said for OSR to count as a genuine alternative to traditional metaphysics. In lieu of further clarification, OSR is mere sloganeering.

\section{Generalism}

One potential reply to the above challenge is provided by generalism, the view advocated by Dasgupta (2009, 2016).8 According to generalism, the most fundamental facts about the world are purely general and qualitative. It contrasts with individualism, which takes as fundamental facts about individuals and their properties $9^{9}$ Roughly speaking, individualism takes as basic facts of the form $F a, G b, R a b$ while generalism takes as fundamental quantified expressions such as $\exists x F x, \exists y G y, \exists x \exists y R x y$. Individualism is an intuitive view; many of us are sympathetic to the thought that (to use the popular metaphor) God laid down the individual facts, and the general facts followed from these. So why does Dasgupta reject individualism?

Very briefly, my reason is that if individualism were true then the individualistic facts of our world would lie beyond our epistemic ken. The idea is that our knowledge of the world is limited to knowledge of its qualitative nature and whatever is grounded in that qualitative nature, and since individualism implies that there are further facts of the matter as to which particular individuals lie behind those qualities it follows that those facts would be unknowable. A reasonable Occamist principle then recommends that we dispense with such epistemically inaccessible facts. (Dasgupta, 2014, 6)

These considerations motivate qualitativism over individualism; fundamental facts should not make reference to any specific individuals. But there are other varieties of qualitativism distinct from generalism. There is, for instance, the traditional bundle theory according to which individuals are reduced to bundles of compresent properties. The bundle theory, however, faces well known objections concerning symmetric situations such as Max Black worlds. An important goal for Dasgupta is to provide a qualitativist view that is capable of describing such symmetric states of affairs. This is where the appeal to general facts

\footnotetext{
${ }^{8}$ Several others have defended versions of generalism and closely-related views, perhaps even Leibniz (Møller-Nielsen, 2016). My concern here is only with the specific version of generalism advocated by Dasgupta.

${ }^{9}$ As a first-pass, Dasgupta takes individuals to be "what in ordinary English we call "things" - e.g. apples, alligators, atoms, and so on." $(2016,1)$. As discussed below, there is some ambiguity in generalism's understanding of individuals (see section 4.2 .
} 
is helpful. If one abandons the aim of recovering individuals (e.g., as bundles of qualitative properties) and instead focuses on describing qualitative states of affairs directly, the problems of the bundle theory can be avoided. For example, suppose we have a Max Black world that contains only two perfectly similar iron spheres some distance apart. The individualistic description has the form: $F a, F b, D a b$, where $F$ is a summary of the intrinsic properties of each iron sphere and $D$ is the distance relation between them. The bundle theorist, it seems, is compelled to identify the "two" iron spheres and thereby reject the original description. Generalism, however, can take the situation at face value and claim that the fundamental fact in question is: $\exists x \exists y(F x \wedge F y \wedge D x y \wedge x \neq y)$. Notice that the generalist must endorse a kind of holism here; the entire state of affairs must be described by a single qualitative fact, not the conjunction of several. The parallels with OSR are clear. Instead of telling us what individuals there are and how they're propertied, generalism provides a qualitative description of the entire situation - only its structure, one might say - without any mention of individual objects. Thus, Dasgupta suggests that generalism can provide just the sort of "worked-out metaphysics" OSR is in need of.

Generalism is a view very much in the ballpark of what [the slogans of OSR] gesture at. If so, it serves as a "proof by construction" that there are coherent views corresponding to the [slogans]. Thus generalism is a natural resource for structural realists keen on developing their views. (Dasgupta, 2016, 14)

There also seems to be some support for generalism among structuralists. For instance, Steven French notes that "[t]here are clear connections with the picture I am sketching here and Dasgupta's 'radical holism' might offer a general (ha!) and congenial home for various forms of structuralism" (French, 2014, 114, n.8). Before we assess the viability of generalism as a form of OSR, it will be useful to distinguish two versions of generalism discussed by Dasgupta: quantifier generalism and algebraic generalism.

\subsection{Quantifier generalism}

Quantifier generalism is the version of generalism sketched above. It takes as fundamental quantified facts that are purely qualitative. For example, $\exists x \exists y R x y$ is taken to be the ungrounded fact ordinarily described as something or other standing in the relation $R$ to something or other. For the individualist, this fact is grounded in there being some individuals $a$ and $b$ such that they bear Rab. That is, individualists would endorse the following general principle:

* Existentially quantified facts are grounded in their instances.

Quantifier generalism is a clear articulation of the generalist approach; it replaces the fundamental individualistic facts with a general, qualitative fact. However, the principle * has considerable intuitive support. Indeed, Dasgupta suggests that it may be "analytic" or 
"essential" of the existential quantifier in some sense (Dasgupta, 2016, 7-8). If that were the case, this way of developing generalism would be untenable; the fundamental facts cannot be existentially-quantified statements of general qualitative states of affairs.

Quantifier generalism also doesn't seem well suited to explicate OSR. Existentially quantified statements, even taken as ungrounded, seem to commit one to the existence of the objects over which they quantify. These objects needn't be the particular individuals $a$ and $b$, but it seems that there must be something or other that stands in relation $R$ to something or other for $\exists x \exists y R x y$ to be true. Perhaps the quantifier generalist can regard the objects in question as non-fundamental, and hence compatible with OSR's insistence that there is only structure at the fundamental level. But, then it becomes increasing hard to comprehend what the world is like at the most fundamental level. How can we make sense of a fact like $\exists x \exists y R x y$ without appealing to the notion of some object(s) standing in relation $R$ ? The main challenge for OSR is moving beyond the slogans to a clearly articulated proposal, but it's hard to see how quantifier generalism can alleviate this worry. Either (a) one recognizes a commitment to objects (generically referenced) or, (b) one endorses a brand of quietism in which we are unable to say anything substantiative about fundamental reality.

If one is happy to reject $*$ and is similarly unbothered by the apparent difficulties just mentioned, then they are free to adopt quantifier generalism. The arguments of section 5 will apply to it. However, for those who do feel the force of these considerations, there is alternative version of generalism available 10

\subsection{Algebraic generalism}

For the generalist, the world consists of a collection of properties (universals) that have a certain structure. The quantifier generalist represents that structure using familiar existentially-quantified statements. The algebraic generalist, by contrast, seeks to capture the structure by introducing an algebra of connectives that relate properties directly. In Dasgupta's system, there are six such connectives, which he calls "term functors." For example, the term functor $\sim$ takes the property $F$ to the complex property "being not $F$ " and $F \& G$ is the property of "being $F$ and $G$." More interesting is the cropping functor $c$ which, intuitively, allows one to say that one place of a given $n$-place property ${ }^{11}$ is filled. Suppose $R$ is a two-place property (relation) we ordinarily describe as "bears $\mathrm{R}$ to." In this case, $c R$ is the one-place property ordinarily described as "something bearing $\mathrm{R}$ to" and $c c R$ is the zero-place property ordinarily described as "something bearing $\mathrm{R}$ to something." One more ingredient is needed to complete the picture. We need some way to say

\footnotetext{
${ }^{10}$ In the next section, I claim that both forms of generalism face a version of the worry just raised. But at a first-pass, moving to the algebraic formulation appears to help avoid the commitment of objects apparently required by quantifier generalism.

${ }^{11}$ In this context properties are broadly construed so as to allow more than one or zero arguments. Thus, properties include what are often regarded as relations and states of affairs.
} 
the 0-place property in question is instantiated. For this purpose, Dasgupta introduces the primitive predicate "obtains." Thus, $c c R$ obtains is the fact ordinarily described as the obtaining of the state of affairs in which something bears $\mathrm{R}$ to something.

To compare the different views, consider a simple example. Suppose that there are two objects, one bigger than the other. Each view offers a different fundamental description of this state of affairs:

Individualist: $B a b$, where $B$ is the "bigger than" relation and $a$ and $b$ name individual objects.

Quantifier generalist: $\exists x \exists y(B x y \wedge x \neq y)$, where $\mathrm{B}$ is the qualitative universal "bigger than" and the entire expression is taken as ungrounded and irreducible.

Algebraic generalist: $c c(B \& \sim I)$ obtains, where $c c B$ is the complex predicate ordinarily described as "something being bigger than something" and $c c \sim I$ is the complex predicate ordinarily described as "something being not identical to something."

Crucially, because the lattermost is non-quantificational, one needn't choose between a commitment to individual facts and denial of the principle $*$.

Before moving on, I wish to flag a certain difficulty in making sense of generalism in either of its forms. On the one hand, its stated ontology is limited to properties; the view seems to eschew property-bearing objects of any sort. However, as noted above, it's hard to make sense of quantifier generalism's ungrounded existential statements without appealing to objects. Even in algebraic generalism, one relies on ordinary descriptions in terms of individuals to give content to the complex predicates involved. Thus, one might wonder whether individuals are being smuggled in even in this version. In the Appendix of his (2009), Dasgupta argues that one can appeal to a domain of individuals to make sense of (and provide a semantics for) generalized statements only to "kick away the ladder" and be left with only the primitive predicates of the algebraic generalist. But, again, one may question how much progress this makes from the slogans of OSR, which also start with something we understand - ordinary set-theoretic structures - and ask us to eliminate the objects. I will leave this worry for now and proceed on the assumption that generalism can sustain an ontology of only properties and no property-bearing objects. If this pure understanding of the view is incapable of serving as an explication of OSR, then so much the worse for an understanding that involves some commitment to objects ${ }^{12}$

\section{Why generalism is not OSR}

There are at least three features of generalism that make it attractive as a potential explication of OSR. First, it doesn't posit individuals as elements of fundamental ontology. Second,

\footnotetext{
${ }^{12}$ I thank an anonymous referee for pressing me on this point.
} 
it is holistic in that it takes as fundamental facts about the entire state of affairs given in purely qualitative terms. Such facts may be naturally thought of as capturing the structure of the world. Third, generalism is anti-haecceitistic; qualitative duplicate worlds are duplicates simpliciter. However, further consideration reveals that none of these features help the structuralist achieve the aim of developing an objectless ontology that captures the motivations discussed in section 2 . Indeed, in some cases they may create additional problems for the structuralist. Of course, there may be other metaphysical systems that fare better, but there is also reason to be skeptical ${ }^{13}$ This merits taking generalism-based OSR seriously and its failure as significant for understanding the structuralist's position.

Consider the first point. In their presentations of OSR, structuralists are often less than precise about the objects they are eliminating. Ladyman and Ross (2007), for instance, sometimes speak of doing away with "self-subsistent individuals" (e.g., 130) but at other times seem to be advocating a more extreme eliminativism. 14 But if OSR is intended to solve the problem of theory change, it must jettison a commitment to the entities that get traditional realists into trouble: things like ether, caloric, phlogiston, etc. And these are not the individuals eliminated by generalism, which are particular individuals that may be distinguished from all of their qualitative features. The generalist version of some scientific theory will involve qualitative features that appear to make reference to the same entities recognized by the traditional realist, it just won't refer to any particular individuals that instantiate them. For instance, the generalized version of Fresnel's optical theory will still make reference to the luminiferous ether qua something with a certain density and vibration state. But, of course, there is nothing which possesses these properties, nor are there states of affairs in which they "obtain".

As a comparison, it's helpful to consider the Ramsey sentence approach to structuralism. On this view, structure is taken to be captured by the Ramsey sentence associated with some theory. If we assume the theory is formulated as a sentence in first-order predicate logic, then the Ramsey sentence of that theory is a second-order formula in which the predicates are replaced by (second-order) variables falling under the scope of a (secondorder) existential quantifier. Proponents of OSR have widely rejected formulating structure in this manner. The Ramsey sentence is both too weak and too strong an understanding of structure for their purposes. It's too weak because any collection of objects with the right cardinality will trivially realize a given Ramsey sentence (Newman's problem). It's too strong because it fails to make a substantive concession to the antirealist; it is still committed to the same entities as the traditional realist, differently described. Given the close parallel between generalism (especially quantifier generalism) and Ramsey sentence realism, one may reasonably worry that these problems will arise in this context as well.

\footnotetext{
${ }^{13}$ At least among extant metaphysical frameworks, there is some reason to agree with Ted Sider that generalism is "the best view, in my perspective, in the vicinity [of OSR]" (2016, Lec. 5). I return to this point in section 7

${ }^{14}$ For example, when they claim "a first approximation to our metaphysics is: "There are no things. Structure is all there is." " 2007, 130)
} 
The analog of Newman's problem would be that any collection of individuals with the right cardinality can be said to realize the generalized description of the world. Of course, Dasgupta rejects that there are any such individuals, so it's not clear that this objection will apply (though it may be a problem for a version of ESR based on generalism). The second problem - that we haven't changed the ontological commitments - is also mitigated by Dasgupta's claim that there are no (facts about) individuals grounding general states of affairs. However, even if we restrict our ontological commitments to purely qualitative properties, there are still problems. First, these properties will often be explicated by appeal to the idea of "something being X" which at least seems to involve a commitment to objects if not individuals in the sense discussed above ${ }^{15}$ Second, even if we can make sense of an ontology of only qualitative properties, it's far from clear that these are any more likely to be preserved across theory change than the objects recognized by traditional realists.

Thus, generalism's rejection of fundamental individuals doesn't seem to help the structuralist. Generalism involves either a commitment to generic objects - something bearing certain qualitative properties - or at least a commitment to the full complement of qualitative properties posited by our best scientific theories. Of course, it may be possible to adopt an attitude of selective skepticism toward certain of these properties in an effort to restore continuity across theory change. However, there doesn't seem to be any special contribution from generalism in such a case; the same move could be made starting from a traditional realist ontology. Generalism, then, doesn't seem to help the structuralist account for the first motivation for OSR - the problem of theory change.

The second aspect of generalism relevant to OSR is its holism. Indeed, some structuralists give central importance to the holism of OSR.

Structural realism is a holism in contrast to an atomism. In atomism, one considers the world as being composed of atoms in the sense of objects that exist independently of one another because they are characterized by intrinsic properties each [has] ("local matters of particular fact, just one little thing and then another", in the words of David Lewis in the citation above). Holism, by contrast, can be conceived as regarding the whole world - or the domain of the world that one considers - as just one object in the last resort. (Esfeld and Lam, 2010, 14)

The holism urged by structuralists - often motivated by quantum entanglement - is an ontological holism. It seeks to regard wholes (e.g., compound entangled systems) as ontologically prior to their parts (e.g., entangled particles). The holism of generalism, by contrast, comes from the fact that complex states of affairs must be described by a single

\footnotetext{
${ }^{15}$ As noted above, Dasgupta $(2009,66)$ claims that this conceptual dependence on objects needn't imply ontological commitment to them, at least in the context algebraic generalism. Whether the commitment to objects is avoided for either version of generalism is, however, not entirely clear.
} 
expression rather than being built up by conjoining more simple expressions. Recall that this is required in order to meet the challenge of accounting for symmetric situations such as Max Black worlds that create problems for other qualitativist views such as the bundle theory. In fact, generalism ultimately leads to a version of monism in which "fundamentally speaking at least, there is only One Great Fact that captures our entire world all at once" (Dasgupta, 2009, 56). This sort of radical holism is more akin to semantic holism than the ontological variety favored by structuralists. This suggests that the holism of generalism may in fact be a liability from the structuralist perspective.

Again it will be useful to recall Ramsey sentence realism. One problem with the view is that it leaves the ontological commitments of the traditional realist untouched, but there is reason to think it might make the situation worse for the structuralist.

The Ramsey sentence picks out exactly the same entities as the original theory. It does not dispense with reference, but it makes that reference a function of the (place of the theoretical terms in the) overall structure of the theory, as manifested in the Ramsey sentence. The problem of ontological discontinuity is left untouched by simply adopting Ramsification. In fact, it seems even worse if contextualism about the meaning of theoretical terms is adopted. (Ladyman, $2009, \S 3.2)$

The problem suggested at the end of the passage is that contextualism (i.e., semantic holism) makes it impossible to regard parts of an abandoned theory as correct. If the meaning of a term depends on the entire theoretical context in which it occurs, preserving meanings across theory change becomes impossible. An analogous problem besets generalism in light of its holism. The fundamental description of the world functions as a single fact, and hence, if it changes, we have an entirely new fact. Because of the holism of these facts, we cannot separate-out parts of this description that are retained or approximated across the change. Preservation and continuity across theory change become an all or nothing affair, as with contextualism. As a result, the structuralist's aim to restore continuity across theory change becomes severely hamstrung by the extreme holism of generalism. The strategies of structuralists - e.g., the partial structures approach of da Costa and French (2003) - cannot be utilized if the description of the world cannot be separated into parts.

This is not to say there is no sense of continuity to be found on the generalist picture. Even if, fundamentally speaking, theory change involves the wholesale replacement of one world fact with another, there could be similarities between these facts. For instance, both facts might entail that $\exists x F x$. But, notice that this is not continuity in the world facts themselves, but rather in their consequences. The same sort of continuity is possible for the semantic holist; even if "mass" has a different meaning in relativity and Newtonian mechanics, these theories may agree in certain of their consequences (e.g., the motions of bodies when speeds are slow and distances short). But, for the realist, this isn't enough. 
Discontinuity with respect to some class $X$ problematizes realist commitment to members of $X$ - this is the essence of the antirealist's pessimistic induction. Antirealists are happy to allow for continuity with respect to some distinct class $Y$ of entailments from $X$, for example, the empirical predictions that follow from members of $X$. In such a case, the antirealist with respect to $X$ can argue that continuity in the class $Y$ means that it is members of this class rather than $X$ that are worthy of endorsement.

In the present context, the upshot is as follows. The holistic theories delivered by generalism aren't the appropriate target of commitment, but rather, one should endorse some of their consequences, namely, those that exhibit the requisite continuity. But, a central motivation for structural realism is to deliver an appropriate target of realist commitment. Thus, generalism needs to demonstrate continuity at the level of the fundamental description of the world given by the scientific theories in question, suitably understood. But generalism cannot provide this because it regards this description as non-compositionalit simply doesn't contain things like $\exists x F x$ as parts. Hence, the most generalism can provide is the same sort of continuity of consequences that is available to the semantic holist and inadequate as a response to the antirealist challenge.

So far, we have focused solely on the first motivation for OSR: the problem of theory change. But, perhaps generalism could be seen as a suitable explication of a version of OSR motivated purely on the basis of the second motivation: permutation invariance in fundamental physics. Indeed, it is thinking about permutations and anti-haecceitism that Dasgupta takes to provide motivation for generalism. However, even in this area it's not clear that generalism delivers what structuralists are after.

Generalism offers a framework in which (putative) permutations cannot be taken to generate distinct states of affairs. Consider two individuals $a$ and $b$ such that $F a$ and $G b$ represent all of their qualitative (monadic) properties. A permutation of these individuals $(F a, G b) \rightarrow(F b, G a)$ is blocked by either variety of generalism; these two expressions are replaced by the single expression $\exists x \exists y(F x \wedge G y \wedge x \neq y)$ or $c c(G \& F \& \sim I)$ obtains. Is this the kind of permutation invariance that is taken to motivate OSR? There is some reason to think not. Note that the permutations in question concern only a very specific class of entities - indistinguishable particles in QM. There is a tradition going back to Born and Heisenberg that takes quantum particles to be "non-individuals" quite unlike familiar objects on the basis of permutation invariance. This implication has been challenged by contemporary philosophers of physics (French and Rickles, 2003; Saunders, 2006), but there remains the question of why permutation invariance occurs in QM and not elsewhere. Structuralists aim to answer this question by taking fundamental physics to concern structures that are invariant under permutations. According to generalism, by contrast, everything is permutation invariant. Thus, the puzzle recurs in a different form: why, if everything is permutation invariant, do non-quantum systems obey classical (Maxwell-Boltzmann) statistics?

For the generalist, there must be some further account of the distinctive variety of permutation invariance displayed by quantum systems. Here it is useful to consider Das- 
gupta's motivation for eliminating individuals, which draws on an analogy with absolute velocity in Newtonian physics:

So if absolute velocity and primitive individuals were real, they would both be undetectable and redundant in the same sense. Just as systems differing only in facts about absolute velocity are indistinguishable, so too are qualitatively identical systems that differ only in which primitive individuals lie behind the qualities. And just as the particular absolute velocities of things make no difference to how a system evolves, so too the particular primitive individuals that populate the system make no difference either. (Dasgupta, 2016, 5)

Hence, for the generalist, undetectability and redundancy are the keys to understanding when permutations matter. This suggests the following strategy for explaining the difference between quantum and classical systems: only in QM is the permutation of indistinguishable particles undetectable and redundant. The permutation of classical particles, by contrast, is presumed to be detectable or non-redundant. It's not clear whether this is a viable strategy. If classical particles are genuinely indistinguishable, how can permuting them be detectable or non-redundant?:16 The important point for present purposes is that even if we grant the generalist this reply, it is not generalism that does the explanatory work, but the notions of undetectability and redundancy. After all, these considerations apply irrespective of whether one is an individualist or a generalist.

Perhaps generalism plays a more limited role in the explanation of permutation invariance ${ }^{17}$ It could be that undetectability and redundancy provides evidence for rejecting certain classes of individuals and generalism provides a framework for doing so. On this view, permutation invariance in QM is evidenced by the undetectability and redundancy of states related by a permutation of particles and explained by the fact that the underlying generalist states of affairs block such permutations. However, given that permutation symmetry is simply built into standard QM, epistemological considerations seem out of place in trying to account for it metaphysically. Moreover, the fact remains that the explanation of permutation invariance should account for what is special about this class of objects such that they, and not other kinds of entities, manifest the invariance. One could maintain that generalism only applies to quantum particles, and not ordinary objects but, regardless of the merits of such a hybrid position, it certainly isn't Dasgupta's view.

Thus, while there are the several candidate explanations of permutation invariance in QM, adopting generalism seems to contribute little to the explanation offered. Indeed, it's

\footnotetext{
${ }^{16}$ Some have claimed that when classical particles are genuinely indistinguishable they no longer obey classical Maxwell-Boltzmann statistics without permutation symmetry. This has been invoked in the explanation of the so-called Gibbs paradox concerning the failure of entropy extensively in classical statistical mechanics. If one adopts this view, then what explains permutation invariance in quantum and classical systems is their indistinguishability, not their lack of "individuality" (Saunders, 2003a, 302).

${ }^{17}$ Thanks to an anonymous referee for suggesting this.
} 
hard to see how the generalist can adopt the received view that takes only quantum particles to be "non-individuals." The alternative explanations of offer - such as the appeal to undetectability and redundancy - rely on distinctive features of indistinguishable quantum particles that are independent of the status of primitive individuals in general. The reason generalism misses the mark here can be traced to the fact that interesting cases of permutation invariance in physics involve more than just the exchange of primitive individuals, which, as Dasgupta correctly observes, is undetectable and redundant for all objects.

\section{OSR as meta-metaphysics}

We've seen that generalism doesn't save OSR from the charge of metaphysical incoherence. While it offers a "worked-out" metaphysics, it is not a metaphysics capable of vindicating the motivations for OSR. In particular, it doesn't license the structuralist replies to the problems of theory change and permutation invariance. What options remain for the advocate of OSR? One option is to retreat to a version of "non-eliminativist" OSR, in which fundamental objects exist, but depend on structure in some sense (e.g., Esfeld and Lam, 2008). Such views face a number of challenges, including the worry of a collapse into traditional realism, but I will not address these here ${ }^{18}$ Instead, I wish to emphasize another strand in thinking about OSR.

In the above, it was assumed that OSR seeks to replace the default "objects bearing properties" view with a rival metaphysical account that consists of only structure. This is somewhat puzzling given the disdain some structuralists express toward analytic metaphysics as standardly conceived 19 Perhaps, then, OSR should be viewed not as metaphysics, but as meta-metaphysics. Consider, for instance, the following remark from Ladyman and Ross.

According to OSR, if one were asked to present the ontology of the world according to, for example, GR one would present the apparatus of differential geometry and the field equations and then go on to explain the topology and other characteristics of the particular model (or more accurately equivalence class of diffeomorphic models) of these equations that is thought to describe the actual world. There is nothing else to be said, and presenting an interpretation that allows us to visualize the whole structure in classical terms is just not an option. Mathematical structures are used for the representation of physical structure and relations, and this kind of representation is ineliminable and irreducible in science. (Ladyman and Ross, 2007, 159)

\footnotetext{
${ }^{18}$ Another worry, discussed below, is that non-eliminativist OSR is not particularly revisionary with respect to metaphysical orthodoxy. For other challenges, see Chakravartty (2012).

${ }^{19}$ Notably, Ladyman and Ross (2007, vii) call for contemporary analytic metaphysics to be discontinued. While they do allow for a variety of naturalistic metaphysics, it is intended to be very different from the sort of activity that characterizes most metaphysics today.
} 
Similar sentiments have been expressed by Saunders (2003b, 2015) and Wallace 2012, $\S 8.8)$. The basic thought is that, following the semantic conception, scientific theories are associated with a collection of mathematical models, and these models themselves provide the most fundamental description of the world. Any attempt to provide a metaphysical picture on the basis of these models will inevitably involve moving to a less perspicuous description. This is not to say that there is no place for metaphysics, nor that all metaphysical accounts are created equal, but rather, that none is capable of providing us with the structure that OSR takes to be fundamental; this requires mathematical representation. On such a view OSR is a meta-metaphysical position in that it concerns the status of metaphysics generally. In particular, it regards any metaphysical account as only an illustration of the world's structure with pragmatic value, but only accurate "for all practical purposes." Saunders (2003b) explicitly endorses such a position, but sees this as a contrast with other structuralists.

French, Ladyman, and I are agreed that the logical notion of object is inadequate to ontology, according to the structuralist, but they see the problem as more severe than do I, and unlike them, I see no reason to seek for an alternative notion of object-hood. The world is a structure, and it is thought of as such in exact physical, interpreted mathematical terms, but how it is to be broken down into parts, to be spoken of predicatively, can be a more rough and ready affair, sufficient only in the sense of FAPP, to use Bell's acronym; sufficient linguistically, but only for all practical purposes. (2003b, 132)

However, some passages from French and Ladyman - such as the Ladyman and Ross passage quoted above - suggest the difference isn't so great. Moreover, given the difficulties that beset OSR when construed as a (first-order) metaphysical position, it may be preferable for structuralists to adopt the meta-metaphysical construal.

Meta-metaphysical positions typically have implications for the practice of metaphysics. For instance, Hirsch's (2009) claim that certain ontological disputes are purely verbal implies that we shouldn't waste our time on them. The implications of meta-metaphysical OSR for the practice of metaphysics are less clear. One might, as Saunders seems to, allow for metaphysics to carry on in its present form with the proviso that it is of only pragmatic value and hence should not claim to be offering a fundamental description of the world. Alternatively, one could take the non-fundamental status of metaphysics to have more revisionary implications for the practice of metaphysics including, as a limiting case, the discontinuation of the discipline advocated by Ladyman and Ross. Other positions, intermediate to these extremes, are possible as well ${ }^{20}$ From the current perspective, it is a disagreement about these implications that is really at issue in Saunders' remark. All

\footnotetext{
${ }^{20}$ For instance, the "toolbox" view advocated by French and McKenzie $(2012,2015)$ allows for work in metaphysics that may have instrumental value in the development of science. Such a view would also fit comfortably with the meta-metaphysical construal of OSR.
} 
versions of OSR, properly so called, should be taken to view mathematical descriptions of structure as more fundamental than metaphysical ones. Where structuralists differ is what this means for the practice of metaphysics.

There is also reason to think that the meta-metaphysical understanding of OSR fits better with the motivations discussed in section 2. The problem with generalism as an explication of OSR is that it fails to license the structuralist positions on theory change and permutation invariance, but the present understanding fares better. First, consider the problem of theory change. The structuralist response - going back to Poincaré and Worrall - is to invoke relations of mathematical continuity. For instance, in the case of Fresnel and Maxwell, it is alleged that one can recover the equations of the former as a special case of the latter. The structuralist claims that such "correspondence" relations are the norm in physics, and hence we should be optimistic about the prospects of current mathematical structures surviving (at least approximately) in this manner. Of course, such a claim is controversial, even in the case of Fresnel and Maxwell. My claim is simply that the meta-metaphysical construal of OSR better licenses this reply, regardless of its merit. For, suppose that we have preservation or continuity of mathematical structure of the relevant sort. The traditional realist cannot (straightforwardly) appeal to this fact because the mathematics occurs in the context of different theories, with different ontological commitments. Similarly, the generalist will associate with each theory some purely qualitative description of the world. Even if the theories are continuous in mathematical structure, there is no reason to think they will be continuous in the variety and distribution of qualitative properties they posit. Hence, on the assumption that mathematical structure is continuous, meta-metaphysical OSR is better able to adopt the standard structuralist reply to the problem of theory change.

Second, consider the explanation of permutation invariance. On the meta-metaphysical construal, the question is what mathematical structure accounts for this feature of indistinguishable quantum systems. One possible answer is given by "reduced configuration space" approach discussed by French and Rickles (2003). If one takes the configuration space associated with a system of many indistinguishable particles to be the $n$-fold Cartesian product of the spaces associated with each of the $n$ particles, then states related by a permutation will be associated with distinct points in the product space. However, one can eliminate the redundant points by taking the quotient of the standard space by the action of the relevant symmetry group (including, in this case, the permutation operation) to arrive at the reduced configuration space. On this view, permutation invariance is built into the topological structure of the reduced space; a permutation of particles labels corresponds to the same point in the space, and hence cannot have physical significance. Does such an approach fare any better than generalism in licensing the structuralist take on permutation invariance?

The structuralist seeks to locate indistinguishable quantum particles in a structure that is invariant under permutations. If the particles (or their "identities") depend on such a structure, then permuting them to generate a distinct state of affairs is impossible. The 
problem for generalism is that it fails to distinguish quantum systems from non-quantum ones; everything is permutation invariant in Dasgupta's sense. The reduced configuration space approach, by contrast, applies only to those systems governed by laws that contain the relevant symmetry ${ }^{21}$ One quotients out only those features that correspond to symmetries of the theory in question, hence only a theory with permutation symmetry will be associated with a reduced state space in which permutations are blocked. The problem with generalism's approach to permutations is not its strength - that it makes permutations of individuals impossible - but rather its scope - that it applies to everything. The meta-metaphysical view, by contrast, allows for OSR to explain permutation invariance in QM by identifying a mathematical structure that represents quantum systems in such a way as to block permutations. The reduced state space approach is one way to arrive at such a structure, but there may be others as well. As long as some such structure is found, the meta-metaphysical view can achieve the structuralist's aim.22

More work is required to develop OSR into a clear and defensible meta-metaphysical position. One looming question concerns the status of the mathematical models to which it appeals. For the distinction between mathematical and metaphysical models to be sustained, one must have an understanding of the former that doesn't appeal to traditional metaphysical categories of objects and properties. Hence, it could be that a defense of this version of OSR would require substantial commitments in the philosophy of mathematics; perhaps one must endorse a version of structuralism in that context as well. Addressing such concerns is important future work for structuralists.

Meta-metaphysical OSR may also require the revision of certain structuralist claims. For instance, is it still true that "there is only structure at the fundamental level"? After all, on the present understanding, OSR doesn't provide a metaphysics, and this appears to be a metaphysical claim. Hence, claims such as this one must be recast in meta-metaphysical terms; what it is for structure to be fundamental is for a description of the world cast in terms of mathematical structure to be privileged as compared to one in metaphysical terms. The resultant revision may seem to move OSR closer to its epistemic counterpart,

\footnotetext{
${ }^{21}$ Again, some have claimed that indistinguishable classical particles also display permutation symmetry. This would mean that they admit of a reduced configuration space treatment as well (see, Huggett (1999); Saunders (2003a, 302)). If one takes this view, some other feature must account for the difference between classical and quantum systems. However, there is still an important difference between indistinguishable particles - quantum and classical - that exhibit permutation symmetry, and other entities that do not exhibit this symmetry. The reduced configuration space approach respects this difference while generalism does not.

${ }^{22}$ Sider discusses similar issues in his Locke lectures (Sider, 2016, Lec. 6). However, what he calls "quotienting" should be distinguished from the reduced configuration space approach. For Sider, quotienting involves the rejection of a single best description in favor of an equivalence classes of descriptions; the quotienter is happy to note that there is a collection of theories related by a primitive equivalence relation and leave it at that. By contrast, the reduced configuration space approach seeks to eliminate surplus structure by moving to a state space in which the symmetry doesn't occur. Because it locates a single structure underlying the equivalent descriptions, it counts as what Sider calls a "fundamentality view" and hence avoids his objections to quotienting.
} 
but we should also bear in mind the argument against ESR from section 2.1, which focused on the gap it posits between epistemology and metaphysics. Meta-metaphysical OSR, like metaphysical OSR, rejects such a gap; the restriction to mathematical structures is not an epistemic limitation, but a reflection of the nature of our world. Thus, while some structuralist claims may need to be revised on the meta-metaphysical view, it remains faithful to the spirit of OSR.

\section{Conclusion}

OSR has struggled to find a coherent formulation. At a first glance, generalism appears to offer just what is needed: a worked-out metaphysical picture that eschews individual objects in favor of purely qualitative and general states of affairs. Unfortunately for the structuralist, closer examination reveals that generalism is unable to capture the core motivations for OSR. There may well be other metaphysical systems that fare better, and indeed, structuralists are eagerly exploring such alternatives. But there may be reason for pessimism as well. Any first-order metaphysical construal of OSR must meet three desiderata: (1) be sufficiently clear to count as a coherent metaphysical proposal; (2) offer a genuine alternative to metaphysical orthodoxy; (3) be genuinely structuralist by capturing the core motivations for OSR. It is exceedingly hard to achieve all of these aims. For instance, the non-eliminativist version of OSR mentioned briefly above restores conceptual clarity by allowing that there are both objects and structures, but tries to deflate the significance of the objects in some way (e.g., by taking them to have only extrinsic properties). However, such a view isn't particular novel or revisionary with respect to traditional metaphysics nor is it particularly structuralist; for instance, there is no a priori reason why such "thin" objects couldn't be permuted. The views expressed by well-known structuralists like French and Ladyman face the opposite problem when viewed as first-order metaphysics. While they appear (at least in their presentation) to be strongly revisionary and structuralist, they aren't sufficiently worked-out to count as a viable alternative to traditional metaphysics. Dasgupta's generalism is unique in that it seems to have the characteristics required to meet all three desiderata 23

The failure of generalism as an explication of OSR, then, provides some motivation to explore an altogether different approach. The meta-metaphysical construal of OSR is such an approach. It is not my intent here to claim either that meta-metaphysical OSR is the correct view of science, nor that I'm the first to discuss it. Rather, my claim is that it represents the best way of understanding OSR. To the extent that one finds the motivations for OSR compelling, they should endorse this construal of the view ${ }^{24}$

\footnotetext{
${ }^{23}$ Sider's remark in footnote 13 is motivated by similar considerations.

${ }^{24}$ Many thanks to Shamik Dasgupta, Thomas Møller-Nielsen, the anonymous referees and audiences at the Oxford TWiP Seminar and the APA Pacific for their helpful comments.
} 


\section{References}

Bain, J. (2011). Category-theoretic structure and radical ontic structural realism. Synthese, $1-15$.

Brading, K. and A. Skiles (2012). Underdetermination as a path to structural realism. In Structural Realism, pp. 99-115. Springer.

Caulton, A. and J. Butterfield (2012). Symmetries and paraparticles as a motivation for structuralism. The British Journal for the Philosophy of Science 63(2), 233-285.

Chakravartty, A. (2012). Ontological priority: the conceptual basis of non-eliminative, ontic structural realism. In E. L. Dean Rickles (Ed.), Structural Realism: Structure, Object, and Causality, Chapter 10, pp. 187-206. Springer.

da Costa, N. C. and S. French (2003). Science and Partial Truth. Oxford: Oxford University Press.

Dasgupta, S. (2009). Individuals: An essay in revisionary metaphysics. Philosophical Studies 145(1), 35-67.

Dasgupta, S. (2014). On the plurality of grounds. Philosopher's Imprint 14(20).

Dasgupta, S. (2016). Quality and structure. In E. Barnes (Ed.), Current Controversies in Metaphysics, pp. Forthcoming. Routledge.

Esfeld, M. and V. Lam (2008). Moderate structural realism about space-time. Synthese 160, $27-46$.

Esfeld, M. and V. Lam (2010). Holism and structural realism. In R. Vanderbeeken and B. D. Hooghe (Eds.), Worldviews, Science and Us: Studies of Analytical Metaphysics. A Selection of Topics From a Methodological Perspective., pp. 10-31. Singapore: World Scientific Publishing.

French, S. (1989). Identity and individuality in classical and quantum physics. Australasian Journal of Philosophy 67(4), 432-446.

French, S. (2014). The Structure of the World: Metaphysics and Representation. Oxford: Oxford University Press.

French, S. and J. Ladyman (2003). Remodelling structural realism: quantum physics and the metaphysics of structure. Synthese 136-141, 31-56.

French, S. and K. McKenzie (2012). Thinking outside the toolbox: towards a more productive engagement between metaphysics and philosophy of physics. European Journal of Analytic Philosophy 8(1), 42-59.

French, S. and K. McKenzie (2015). Rethinking outside the toolbox: Reflecting again on the relationship between philosophy of science and metaphysics. In T. Bigaj and C. Wüthrich (Eds.), Metaphysics in Contemporary Physics, pp. 25-54. Brill. 
French, S. and M. Redhead (1988). Quantum physics and the identity of indiscernibles. The British Journal for the Philosophy of Science 39(2), 233-246.

French, S. and D. Rickles (2003). Understanding permutation symmetry. In K. Brading and E. Castellani (Eds.), Symmetries in Physics: Philosophical Reflections, pp. 212-238. Cambridge: Cambridge University Press.

Glick, D. (2015). Minimal structural essentialism. In A. Guay and T. Pradeu (Eds.), Individuals Across the Sciences, pp. 207. Oxford University Press.

Hirsch, E. (2009). Ontology and alternative languages. In D. J. Chalmers, D. Manley, and R. Wasserman (Eds.), Metametaphysics: New Essays on the Foundations of Ontology, pp. 231-58. Oxford University Press.

Huggett, N. (1999). Atomic metaphysics. The Journal of Philosophy 96(1), 5-24.

Kuhn, T. (1962/1996). The Structure of Scientific Revolutions (Third ed.). Chicago: The University of Chicago Press.

Ladyman, J. (1998). What is structural realism? Studies In History and Philosophy of Science Part A 29(3), 409-424.

Ladyman, J. (2009). Structural realism. In E. N. Zalta (Ed.), The Stanford Encyclopedia of Philosophy (Summer 2009 ed.).

Ladyman, J. and D. Ross (2007). Every Thing Must Go: Metaphysics Naturalized. Oxford: Oxford University Press.

Lam, V. and C. Wüthrich (2015). No categorial support for radical ontic structural realism. The British Journal for the Philosophy of Science 66(3), 605-634.

Laudan, L. (1981). A confutation of convergent realism. Philosophy of Science 48(1), $19-49$.

Møller-Nielsen, T. (2016). Was Leibniz a generalist? Studia Leibnitiana 47(1), 8-43.

Pooley, O. (2006). Points, particles, and structural realism. In D. Rickles, S. French, and J. Saatsi (Eds.), The Structural Foundations of Quantum Gravity, pp. 83-120. Oxford: Oxford University Press.

Psillos, S. (2006). The structure, the whole structure, and nothing but the structure? Philosophy of Science 73(5), 560-570.

Saatsi, J. (2005). Reconsidering the Fresnel-Maxwell theory shift: How the realist can have her cake and EAT it too. Studies in History and Philosophy of Science Part A 36(3), $509-538$.

Saunders, S. (2003a). Physics and leibniz's principles. In K. Brading and E. Castellani (Eds.), Symmetries in physics: Philosophical reflections. Cambridge University Press.

Saunders, S. (2003b). Structural realism, again. Synthese 136(1), 127-133.

Saunders, S. (2006). On the explanation for quantum statistics. Studies In History and 
Philosophy of Science Part B 37(1), 192 - 211.

Saunders, S. (2015). On the emergence of individuals in physics. In A. Guay and T. Pradeu (Eds.), Individuals Across the Sciences, pp. 165 d. Oxford University Press.

Sider, T. (2016). John Locke Lectures. Oxford, UK: University of Oxford. Available online at http://www.philosophy.ox.ac.uk/john-locke-lectures.

Stachel, J. (2002). The relations between things versus the things between relations: the deeper meaning of the hole argument. In D. B. Malament (Ed.), Reading Natural Philosophy/Essays in the History and Philosophy of Science and Mathematics, pp. 231-266. Chicago and LaSalle, IL: Open Court.

Wallace, D. (2012). The emergent multiverse: Quantum theory according to the Everett interpretation. Oxford: Oxford University Press.

Worrall, J. (1989). Structural realism: the best of both worlds? Dialectica 43(1-2), 99-124. 